\title{
Über die Einwirkung von Ammoniak auf die Lactone
}

von

\author{
Dr. Hans Meyer.
}

Aus dem chemischen Laboratorium der k. k. deutschen Universität in Prag.

(Vorgelegt in der Sitzung am 13. Juli 1899.)

Die classischen Arbeiten von Fittig ${ }^{1}$ haben erwiesen, dass bei der Einwirkung wässerigen oder alkoholischen Ammoniaks auf die Lactone Additionsproducte entstehen, denen nach diesem Forscher die Constitution von Oxysäureamiden:

$$
\left\{\begin{array}{l}
\mathrm{C}-\mathrm{OH} \\
\mathrm{CONH}
\end{array}\right.
$$

zukommt, während Anschüt $z^{2}$ denselben aldehydammoniakähnliche Configuration:

zuschreibt.<smiles>CC1(N)CCCC1(O)O</smiles>

Die Entscheidung im Sinne der Fittig'schen Auffassung hat bekanntlich erst vor Kurzem Gustav Kramer ${ }^{3}$ zu erbringen vermocht.

Wir müssen also annehmen, dass die Einwirkung wässerigen, beziehungsweise alkoholischen Ammoniaks auf die Lactone, das heisst auf die inneren Säureester analog der

1 Ann., 256, 147 etc.

2 Ann., 259, 143.

3 Ber., 31, 2813. 
Reaction mit den äusseren, den Alkylestern verläuft, indem der Lactonring aufgespalten und der Brückensauerstoff an der Carboxylgruppe durch die primäre Amingruppe substituirt wird, während anderseits ein alkoholisches, beziehungsweise phenolisches Hydroxyl sich bildet.

Die von Fittig untersuchten Lactone zeigten alle die Eigenthümlichkeit, bei höherer Temperatur ihr Ammoniak quantitativ wieder abzuspalten, unter Regeneration des Lactons, und ebenso verhalten sich zahlreiche, in der aromatischen Reihe seither aufgefundene Lactone.

Andere derartige Ringsysteme können überhaupt nicht oder nur unter ganz besonderen Bedingungen mit $\mathrm{NH}_{3}$ zur Reaction gebracht werden, und schliesslich gibt es Substanzen dieser Kategorie, welche direct oder durch Wasserabspaltung aus wenig beständigen Oxysäureamiden Lactame (Imide) liefern.

Wie allenthalben in der organischen Chemie, zeigt es sich auch bei der Ammoniakreaction der Lactone, dass innerhalb einer und derselben Gruppe von Substanzen die Bildung einer bestimmten, typischen Reaction einmal stattfindet, dann wieder, bei einem nahen Derivate des untersuchten Körpers, ohne äusserlich erkennbare Ursache ausbleibt.

Die anscheinende Regellosigkeit im Verhalten der einzelnen Lactone macht indessen einer strengen Gesetzmässigkeit Platz, wenn man die verschiedenen in Betracht kommenden vier-, fünf- und sechsgliedrigen Ringsysteme kritisch durchmustert.

Bei der Einwirkung von Ammoniak in wässeriger oder alkoholischer Lösung findet entweder

1. überhaupt keine Einwirkung auf das Lacton statt,

2. oder es entsteht ein Oxysäureamid, welches leicht das Lacton regenerirt, oder

3. das primär entstandene Oxysäureamid geht mehr oder weniger leicht durch blosses Umkrystallisiren, Erwärmen oder Digeriren mit Alkalien oder Säuren unter Wasserabspaltung in ein Imid (Lactam) über.

Im Folgenden sind nun vorerst die wichtigsten Lactone, nach Ringsystemen geordnet, aufgeführt und den Literaturangaben gemäss ihr Verhalten gegen Ammoniak skizzirt. 


\section{Erstes Schema:}<smiles>CC1(C)C(=O)C(C)(C)C1(C)C</smiles>

In diese Gruppe gehören die von Fittig, Neugebauer und Hjelt untersuchten Fettsäurelactone:

1. $\gamma$-Valerolacton ${ }^{1}$<smiles>CC1CCC(=O)O1</smiles>

liefert mit wässerigem und alkoholischem $\mathrm{NH}_{3}$ leicht $\gamma$-Oxyvalerianamid, das beim Erhitzen auf $180^{\circ}$ wieder quantitativ das Lacton regenerirt.

2. $\gamma$-Caprolacton ${ }^{2}$<smiles>CCC1CCC(=O)O1</smiles>

verhält sich ganz analog, ebenso das

3. Phenyl- $\gamma-O x y b u t t e r s a ̈ u r e l a c t o n^{3}$<smiles>O=C1CCCCC1</smiles>

Anders verhalten sich die ebenfalls diesem Schema einzureihenden, von Kühling ${ }^{4}$ dargestellten Nitril- und Carbon-

1 Fittig und Prasch, Ann., 256, 149; Ber, 17, 202. - Neugebauer, Ann., 227, 97.

2 Fittig und Dubois, Ann., 256, 152. - Vergl. Hjelt, Ber. 15. 617.

3 Fittig und Morris, Ann., 256, 155.

4 Ber. 22, 2346; Ber., 23, 708. 
säurederivate der Lävulinsäure, z. B. das Oxynitril der Äthyllävulinsäure:<smiles>CCOCCC(C)(C)O</smiles>

welches leicht in das beständige Methylpyrrolidonsäurenitril:<smiles>CC1(C)CCC(=O)N1</smiles>

übergeht, wenn Ammoniak bei Wasserbadtemperatur auf den Ester einwirkt.

5. Auch Hydrochelidonsäureanhydrid ${ }^{1}$<smiles>O=C1CCC2(OCC(=O)O2)OO1</smiles>

liefert mit alkoholischem Ammoniak beim Eindampfen unter Wasserabspaltung ein beständiges Diimid:<smiles>COC(=O)NC1CCC(=O)CC1</smiles>

\section{Zweites Schema:}<smiles>CC1OC2C(C)C1C2(C)C</smiles>

6. Triphenylcrotolacton ${ }^{2}$

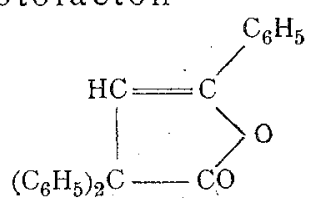

1 Volhard, Ann., 267, 59.

2 Japp und Klingemann, Ber., 22, 2880. - J. ch. soc,, 1890, I, 662. 
und 7. Tetraphenylcrotolacton ${ }^{1}$

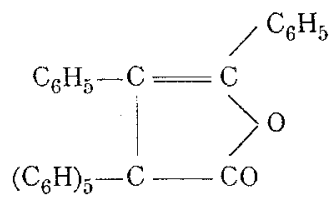

gehen unter dem Einflusse von alkoholischem Ammoniak in 3-Dipheny1-5-Phenylpyrrolon, beziehungsweise Tetraphenylpyrrolon über.

8. Oxydiphenylessigsäurelacton ${ }^{2}$ hingegen

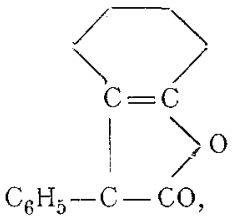

sowie 9. Oxydiphenylbromessigsäurelacton, ${ }^{3}$ weiters

10. Phenylparakresylessigsäurelacton ${ }^{4}$

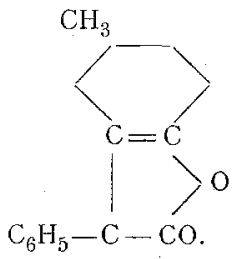

11. Phenylparakresylbromessigsäurelacton, ${ }^{4}$

12.Phenylparakresyläthoxyessigsäurelacton ${ }^{4}$ und

13. Phenylmetakresylessigsäurelacton ${ }^{5}$ liefern mit wässerigem $\mathrm{NH}_{3}$ unbeständige Oxysäureamide, die durch Erhitzen und Einwirkung von Säure das addirte Ammoniak wieder quantitativ abspalten.

1 Klingemann, Ber. 24, 515 .

2 Kramer, Ber. 31, 2814.

3 L. c. S. 2815 .

4. S. 2817 .

5 S. 2820 . 


\section{Drittes Schema:}<smiles>CC1=C(C)C(C)(C)C(=O)C1C</smiles>

Hierher gehört das Phtalid und seine Derivate, die Phtaleïne. 14. Das Phtalid ${ }^{1}$<smiles>O=C1OCc2ccccc21</smiles>

liefert mit alkoholischem Ammoniak in der Kälte ein in grossen glänzenden Blättern krystallisirendes Oxysäureamid, das durch Erhitzen oder Schütteln mit Salzsäure oder Alkali sofort Phtalid regenerirt. Wird hingegen Phtalid mit Chlorzinkammoniak oder im $\mathrm{NH}_{3}$-Strome auf den Siedepunkt (290') erhitzt, so entsteht nach Graebe ${ }^{2}$ Phtalimidin.

15. Phtalylessigsäure ${ }^{3}$<smiles>O=C(O)/C=C1/OOC(=O)C2CC=CC12</smiles>

gibt nach dem Lösen in wässerigem Ammoniak nach dem Ansäuern Phtalimidylessigsäure, und analog bildet sich durch Erwärmen mit verdünntem $\mathrm{NH}_{3}$ aus

16. Phtalylpropionsäure<smiles>O=C(O)CC=C1OOC(=O)c2ccccc21</smiles>

nach Roser $^{4}$ die Phtalimidylpropionsäure.

1. Vergl. auch Bredt, Ann., 286, 352, Anmerkung.

2 Ber., 17, 2598.

3 Gabriel und Michael, Ber., 10, 1556. - Roser, Ber., 17, 2623.

4 Ber., 18, 3120. 
Von den Phtaleïnen ist das Phenolphtaleïn selbst ${ }^{1}$ und das Hydrochinonphtaleïn ${ }^{2}$ mit Ammoniak condensirt worden.

17. Phenolphtaleïn

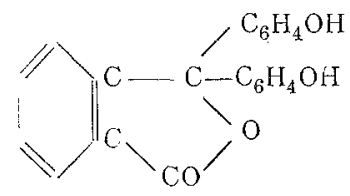

geht, wie ich gezeigt habe, schon beim Stehen mit wässerigem $\mathrm{NH}_{3}$ bei Zimmertemperatur in Imidophenolphtaleïn über.

18. Hydrochinonphtaleïn<smiles>O=C(CO)CC1OOC(=O)C2CCCCC12</smiles>

geht durch kurzes Aufkochen mit concentrirtem wässerigen $\mathrm{NH}_{3}$ in das entsprechende Lactam über.

19. Benzylidenphtalid ${ }^{3}$

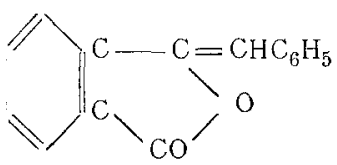

wird durch alkoholisches Ammoniak bei $100^{\circ}$ in Desoxybenzoinsäureamid<smiles>NC(=O)C(C(=O)OCc1ccccc1)c1ccccc1</smiles>

verwandelt, das schon beim Umkrystallisiren aus Eisessig unter vorhergehender Enolisirung und Wasserabspaltung in Benzolphtalimidin übergeht.

1 Hans Meyer, Monatshefte für Chemie, 20, 358, - Vergl. Errera und Gasparini, Gaz. chim. it., 24, I, 75.

2 R. Meyer, Ber., 28, 2961.

3 Gabrie1, Ber., 18, $1257,2434$. 
Ganz analog verhält sich das von Freund ${ }^{1}$ studirte 20. Methylhydrastin

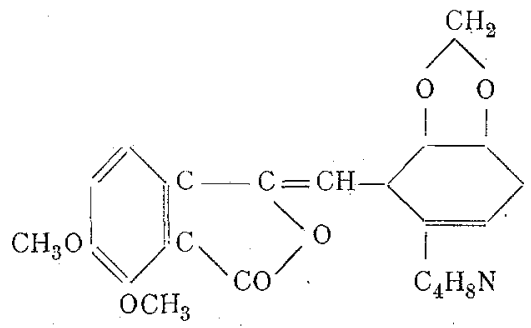

und das homologe

21. Athylhydrastin und 22. Allylhydrastin. ${ }^{2}$

Aus dem intermediär entstandenen Amid wird beim Behandeln mit Säuren oder Basen nach der Umlagerung (Enolisirung) unter Wasserstoffaustritt das Imid gebildet:

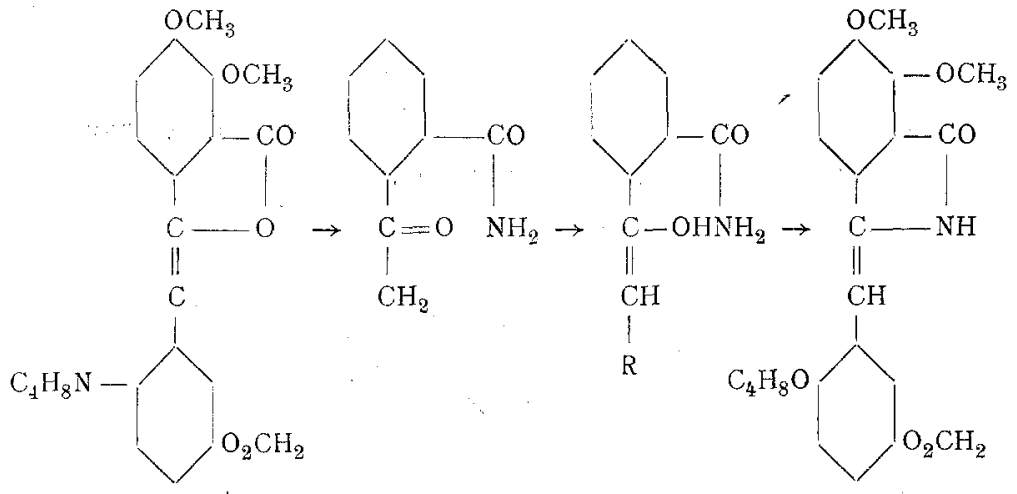

23. Benzaldiphenylmalë̈ ${ }^{3}$<smiles>c1ccc(-c2ccccc2)cc1</smiles>

bildet beim Erhitzen mit alkoholischem $\mathrm{NH}_{3}$ auf dem Wasserbade nach $\mathrm{G}$. Cohn das Amid $\mathrm{C}_{23} \mathrm{H}_{19} \mathrm{NO}_{2}$, das schon beim

1 Ber., 23, 2908.

2 Ber., 23, 2915.

3 Ber., 24, 3059. 
Eintragen in heissem Eisessig in Benzaldiphenylmaleïnidin übergeht.

Das entsprechende Metaxylalimidin bildet sich nach Gysac ${ }^{1}$ direct, wenn man

24. Metaxylaldiphenylmaleid:

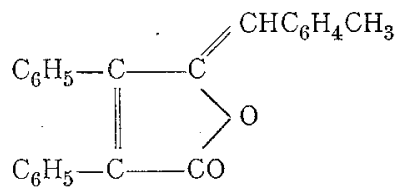

vier Stunden mit alkoholischem zehnprocentigen $\mathrm{NH}_{3}$ bei Wasserbadtemperatur digerirt.

Das analog constituirte:

25. Metaxylalphtalid ${ }^{2}$

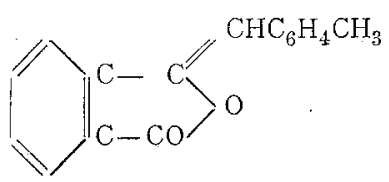

wird schon bei dreistündiger Digestion quantitativ in Metaxylalphtalimidin umgewandelt.

26. Orthoxylalphtalid ${ }^{3}$ gibt nach Fritz Bethmann mit Ammoniak bei $100^{\circ}$ das Oxyamid $\mathrm{NH}_{2} \mathrm{COC}_{6} \mathrm{H}_{4} \mathrm{COCH}_{2} \mathrm{C}_{6} \mathrm{H}_{4} \mathrm{CH}_{3}$, das sich beim Lösen in siedendem Eisessig in Orthoxylalphtalimidin und Wasser spaltet.

27. Cinnamylidendimethylcrotonlacton<smiles>Cc1c(C)c2oc1=CC=CC=2</smiles>

hat vor Kurzem Thiele ${ }^{4}$ erhalten. Es geht beim Erwärmen mit methylalkoholischem Ammoniak in ein Oxysäureamid über, das von Säuren schon in der Kälte und ebenso beim Erhitzen auf $130^{\circ}$ in das Imid $\mathrm{C}_{15} \mathrm{H}_{15} \mathrm{NO}$ übergeführt wird.

1 Ber., 26, 2482.

2 Gabrie1, Ber., 23, 3161.

3 Ber., 32, 1104.

4 Ann., 306, 245. 


\section{Viertes Schema:}

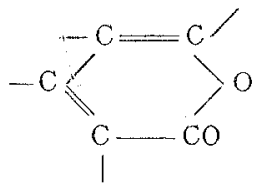

28. Mesitenlacton ${ }^{1}$<smiles>CC1=CC(=O)C=C1C</smiles>

geht schon mit wässerigem Ammoniak in der Kälte langsam in Pseudolutidoshyril über, ebenso wie

29. Isodehydracets äureäther<smiles></smiles>

leicht in Carboxäthylpseudolutidostyril übergeht.2

30. Cumalins äure ${ }^{3}$<smiles>O=C1C=CC(=O)C(C(=O)O)=C1</smiles>

und deren Äther verwandeln sich schon beim Lösen in kaltem wässerigen $\mathrm{NH}_{3}$ in Oxynicotinsäure, wo möglich noch leichter zeigt diese Reaction die ebenfalls von Pechmann studirte

31. Bromcumalinsäure. ${ }^{4}$ Das

32. Phenylcumalin 5

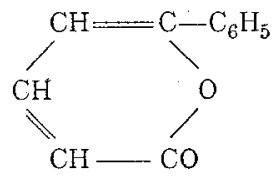

1 Anschütz, Bendix und Kerp, Ann., 259, 168.

2 L. c. 173.

3 Ber., 17, 2390. - Ann., 264, 276.

4 Ber., 17, 2398.

5 Ber., 29, 1677. 
hat J. A. Leben durch Kochen mit essigsaurem Ammon in Eisessiglösung in $\alpha$-Phenyl- $\alpha$-Pyridon verwandelt.

33. Äthoxylpyrondicarbonsäureester ${ }^{1}$<smiles>CCCOC(=O)C1C=CC(C(=O)OCC)CO1</smiles>

geht äusserst leicht beim Lösen in verdünntem Ammoniak in der Kälte in das entsprechende Pyridinderivat über.

Im Gegensatze zu den eben besprochenen Lactonen reagiren die Cumarine im Allgemeinen nicht mit Ammoniak.

34. Cumarin²

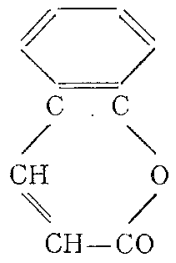

ist nach Gnehm selbst gegen Chlorzinkammoniak resistent.

35. Orthocumarincarbonsäureanhydrid ${ }^{3}$

bildet das Amid

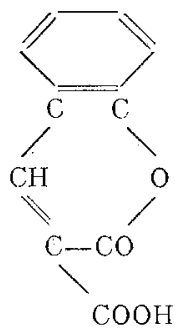

$$
\mathrm{C}_{6} \mathrm{H}_{4}\left\langle\begin{array}{l}
\mathrm{O}-\mathrm{CO} \\
\mathrm{CH}=\mathrm{C}-\mathrm{CONH}_{2}
\end{array}\right.
$$

ohne dass der Lactonring reagiren würde.

1 Guthzeit und Dressel, Ber, 22, 1413. - Ann., 262. 99.

2 Ber., 14, 262.

3 Bechert, J. pr. [2], 50, 27. 
36. Orthonitrocumarin ${ }^{1}$

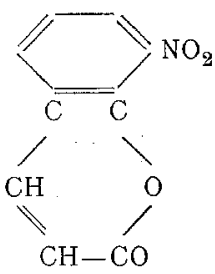

hingegen lässt sich, wenn auch nicht glatt, in Orthonitrocarbostyril überführen.

Die den Cumarinen ähnlich constituirten Isocumarine reagiren nun wieder sehr leicht mit Ammoniak.

37. Isocumarinsäure ${ }^{2}$

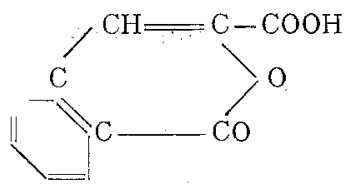

z. B. geht schon in Berührung mit kaltem wässerigen Ammoniak quantitativ in Isocarbostyril über.

38. Isobenzalphtalid ${ }^{3}$<smiles></smiles>

gibt mit alkoholischem und wässerigem $\mathrm{NH}_{3}$ bei Wasserbadtemperatur 3-Phenylisocarbostyril; analog erhält man aus

39. Is oxylalphtalid ${ }^{4}$

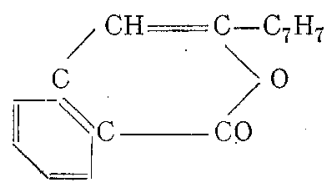

Tolylisocarbostyril, und aus
1 W. v. Miller und Kinkelin, Ber., 22, 1705.
2 Bamberger und Kitsche1t, Ber, 25, 1142.
3 Gabrie1, Ber., 18, 2447, 3471.
4 Heilmann, B. 23, 3167. 
40. 3 -Methylisocumarin ${ }^{1}$

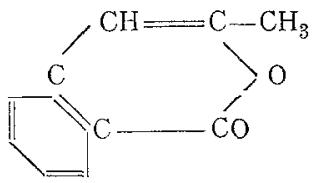

entsteht 3-Methylisocarbostyri1.

41. Orthotolylisocumarin ${ }^{2}$ gibt mit alkoholischem $\mathrm{NH}_{3}$ bei $100^{\circ}$ Orthotoly1-3-Isocarbostyril.

\section{Fünftes Schema:}<smiles>CC1(C)COC1(C)C</smiles>

Die hierhergehörigen $\beta$-Lactone sind nicht in grosser Anzahl bekannt.

42. $d$-Mannozuckersäuredilacton ${ }^{3}$<smiles>O=C1OC(C2OC(=O)C2O)C1=O</smiles>

liefert mit kaltem wässerigen Ammoniak ein Diamid, das sich schon beim Erhitzen auf $189^{\circ}$ und beim Erwärmen mit Alkalien unter Ammoniakentwicklung zersetzt.

43. l-Mannozuckersäuredilacton ${ }^{4}$ bildet ein entsprechendes Oxydiamid, das unter völliger Zersetzung bei $183^{\circ}$ schmilzt und von Kalilauge rasch verseift wird.

44. Ebenso verhält sich. das $i$-Manozuckersäuredilacton. ${ }^{5}$

45. Orthonitrophenylmilchsäure- $\beta$ - Lacton ${ }^{6}$<smiles>O=[N+]([O-])c1ccccc1</smiles>

1 Gabriel, Ber., 25, 3566.

2 Bethmann, Ber., 32, 1110.

3 E. Fischer, Ber., 24, 543.

4 Kiliani, Ber., 20, 2711. - E. Fischer a. a. O. 545.

5 E: Fischer und Smith, Ber., 24, 545.

${ }^{6}$ Basler, Ber., 17, 1495. - Einhorn, Ber., 17, 2013. 
liefert mit Ammoniak in der Kälte das Amid

$$
\mathrm{C}_{6} \mathrm{H}_{4} \mathrm{NO}_{2} \mathrm{CHOHCH} \mathrm{CONH}_{2} \text {, }
$$

das leicht von Säuren und Alkalien unter Ammoniakabspaltung zerstört wird. Ebenso verhält sich das

46. Paranitrophenylmilchsäure- $\beta-L a c t o n,{ }^{1}$ sowie das

47. $\beta$-Lactón der Paraisopropylorthonitrophenylmilchsäure ${ }^{2}$<smiles>O=[N+]([O-])CCCCCCCCCCCO</smiles>

das schon von kalter Natronlauge unter Grünfärbung und $\mathrm{NH}_{3}$-Entwicklung zersetzt wird.

48. Lactonder $p y-1-$ Chinoly $1-\beta-O x y$ propionsäure ${ }^{3}$

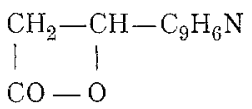

bildet mit kaltem wässerigen und alkoholischen Ammoniak $p y$-1-Chinolyl- $\beta$ Lactamid, das leicht zur Chinolyl- $\beta$-Milchsäure verseift wird.

49. Metachlororthonitrophenylmilchsäurelacton ${ }^{4}$<smiles>O=[N+]([O-])CCCCCCCCO</smiles>

bildet mit wässerigem Ammoniak in der Kälte ein Oxyamid, das sich, auf $150^{\circ}$ erhitzt, zersețzt. Dieselben Forscher haben $o$-Nitrometamethoxyphenyl- $\beta$-Lactamid aus dem

50. $\beta$-Lacton der 0 -Nitro-m-Methoxypheny1- $\beta$ Milchsäure ${ }^{5}$<smiles>COCCCCCCCOC</smiles>

1 Basler a. a. O.

2 Einhorn, Ber.. 17, 2023.

3 Einhorn und Lehnkering, Ann., 248, 175.

4 Eichengrün und Einhorn, Ann., 262, 159.

5 A. a. O. 176. 
erhalten, das beim Anwärmen mit verdünnter Schwefelsäure glatt verseift wird.

Ganz anders als die eben erwähnten $\beta$-Lactone, welche keine Imidoverbindung $\mathrm{zu}$ liefern im Stande sind, verhält sich das

51. Cantharidin ${ }^{1}$

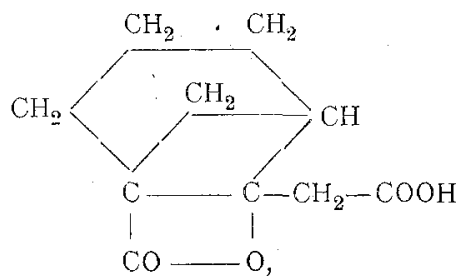

welches beim Eindampfen mit wässerigem oder alkoholischem Ammoniak quantitativ in das Imid

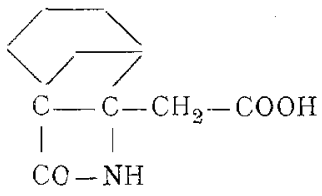

übergeht, das bei $197^{\circ}$ schmilzt und sich unzersetzt destilliren lässt.

Wenn wir die stattliche Reihe der angeführten Lactone überblicken, so zeigt es sich vor Allem, dass die Fähigkeit der Lactambildung sich gleichermassen bei $\beta$-, $\gamma$ - und $\delta$-Lactonen findet, und zwar bei allen vorkommenden Schematis.

Es kann daher die Anzahl der ringbildenden Kohlenstoffe für die Frage der Imidbildung nicht von Belang sein.

Das Verhalten dereinzelnen Lactone gegen Ammoniak ist vielmehr einzig und allein vom Charakter des die Hydroxylgruppe in der zugehörigen Oxysäure tragenden Kohlenstoffatoms abhängig, und zwartritt Imidinbildung mit wässerigem oder alkoholischem oder sonstwie gelöstem $\mathrm{NH}_{3}$ ein:

1 Über dieses Imid, dessen ich schon (Monatshefte für Chemie, 18, 408) Erwähnung gethan habe, werde ich binnen Kurzem ausführlich berichten. 
1. Wenn das Hydroxyl tertiär ist:

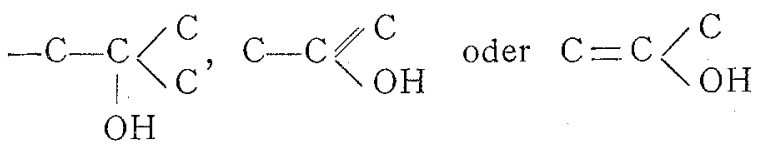

2. Wenn dasselbe secundär und ungesätigt ist:

$$
\mathrm{C}=\mathrm{C}<\begin{aligned}
& \mathrm{H} \\
& \mathrm{OH}
\end{aligned} .
$$

Die Reaction führthingegen bloss zu einem mehr oder weniger labilen Oxysäureamid oder bleibtganz aus, wenn

3. das Hydroxyl einem primären Alkoholrest angehört:

$$
-\mathrm{CH}_{2}-\mathrm{OH} \text {, }
$$

4. oder einem gesättigten secundären Alkohol:

oder endlich<smiles>CCO</smiles>

\section{Phenolcharakter besitzt.}

Wenn sich in Orthostellung zum Phenolhydroxyl eine Nitrogruppe befindet, so kann ebenfalls eine Reaction erzwungen werden, analog wie im Orthonitropheno ${ }^{1}$ schon bei verhältnissmässig niedriger Temperatur directe Substitution des Hydroxyls durch den Ammoniakrest stattfindet.

Die aufgestellten Regeln gelten, soweit ein flüchtiger Uberblick über die Literatur ein Urtheil gestattet, ausnahmslos.

Die nächstliegende Frage, das Verhalten der verschiedenen Gruppen von Alkoholen gegen Ammoniak zu studiren, habe ich bereits in Angriff genommen.

Über die directe Substitution alkoholischen Hydroxyls durch $\mathrm{NH}_{3}$ haben anscheinend nur $\mathrm{Mer} z$ und Gasiorowsky ${ }^{2}$

\footnotetext{
1 Merz und Ris, Ber., 1886, 1751.

2 Ber., 17, 623 .
} 
vor nunmehr 15 Jahren gearbeitet: Die von ihnen untersuchten primären und gesättigten Alkohole reagirten nur mit Chlorcalcium- oder Chlorzinkammoniak bei sehr hoher Temperatur, ebenso wie nach Graebe ${ }^{1}$ das Phtalid. Ein einziger ungesättigter Alkohol wurde bis jetzt mit Ammoniak zur Reaction gebracht, das Phloroglucin<smiles>O=C(O)C1C(=O)CCC(O)C1O</smiles>

das seine Alkoholnatur bekanntlich auch bei der Bildung des Diäthyläthers ${ }^{2}$ durch Salzsäure und Alkohol documentirt und nach den schönen Untersuchungen von J. Pollak ${ }^{3}$ schon in der Kälte durch Ammoniak zweifach substituirt wird.

Auf die vielen Betrachtungen, zu denen die angeführten Substitutionsregelmässigkeiten anregen, bereits jetzt einzugehen, erscheint mir verfrüht, doch wird es mir hoffentlich möglich sein, binnen Kurzem auf die Reactionen zwischen Ammoniak, beziehungsweise den Aminen und dem Sauerstoff, namentlich der Hydroxylgruppe, zurückzukommen.

1 A. a. $\mathrm{O}$.

2 Will und Albrecht, Ber., 17, 2106.

3 Monatshefte für Chemie, 14, 401 ; vergl, auch Hlasiwetz, Ann., 119,202 . 